\title{
Tuberculosis y vitamina D: una relación intrigante
}

\author{
Tuberculosis and Vitamin D: an Intriguing Relationship
}

\author{
Ramírez-Ramos $\mathrm{CF}^{1-2^{*}}$, Salamanca-Montilla JF $\mathrm{F}^{1-2}$, Correa $\mathrm{S}^{1-4}$, Torres-Restrepo JM $\mathrm{M}^{1-4}$, \\ Ramírez-Méndez DA ${ }^{1-4}$, Rivera-Marín $J D^{1-3}$, Pinzón-Tovar $A^{1-5}$, Lastra-González $G^{1-6}$
}

\begin{abstract}
${ }^{1}$ Semillero de investigación en medicina interna -SIMI, grupo de investigación MI-Dneuropsy, Universidad Surcolombiana, Neiva, Huila.

${ }^{2}$ Residente de tercer año de medicina interna, Universidad Surcolombiana, Neiva, Huila.

${ }^{3}$ Médico rural de investigación, Hospital Universitario Hernando Moncaleano Perdomo, Neiva, Huila.

${ }^{4}$ Médico Universidad Surcolombiana, Neiva, Huila.

${ }^{5}$ Médico internista endocrinólogo, coordinador de la clínica de heridas y trastornos metabólicos, Hospital Universitario Hernando Moncaleano Perdomo, Neiva, Huila. Director científico ENDHO Colombia.

${ }^{6}$ Médico internista neumólogo, jefe del servicio de neumología, Hospital Universitario Hernando Moncaleano Perdomo. Jefe departamento de medicina interna, Universidad Surcolombiana, Neiva, Huila.
\end{abstract}

*Correspondencia:

cristhianramos1989@hotmail.com,cfrr89@gmail.com

Fecha de recepción: 27/08/2018

Fecha de aceptación: 28/01/2019

\section{Resumen}

La tuberculosis permanece como la enfermedad infecciosa que más muertes ha causado a lo largo de la historia de la existencia humana, sin poderse aún lograr su control.

En años recientes ha emergido un cúmulo importante de evidencia clínica acerca de la función de la vitamina D (calcitriol o 1,25 hidroxivitamina D) en un papel que va más allá de la homeostasis del calcio. Su papel en el sistema inmunológico es muy interesante vinculando su déficit como un factor de riesgo contribuyente y así mismo fisiopatológico en un gran número de enfermedades autoinmunes e infecciosas. Dentro de éstas se destaca la tuberculosis, enfermedad endémica en nuestro país y en la cual el déficit de dicho compuesto (vitamina D) crea un factor de riesgo para desarrollar esta enfermedad en su forma activa.

Presentamos una revisión detallada de los estudios que explican el papel de la vitamina D en la susceptibilidad para el desarrollo de tuberculosis y los resultados de los estudios clínicos de suplencia de la misma publicados a la fecha.

Palabras clave: tuberculosis, calcitriol.

\section{Summary}

Tuberculosis remains the infectious disease that has caused more deaths throughout the history of human existence, without being able to even manage to control this disease.

In recent years, an important body of clinical evidence has emerged about the role of vitamin D (calcitriol or 1,25 hydroxyvitamin $D$ ) in a role that goes beyond calcium homeostasis; of these its role in the immune system is very interesting, linking its deficit as a risk factor, contributor and physiopathological in a large number of autoimmune and infectious diseases. Within these stands out the tuberculosis, endemic disease in our country and where the deficit of this compound (vitamin D) creates a risk factor to develop this disease in its active form.

We present a detailed review of the studies that explain the role of vitamin $D$ in susceptibility to the development of tuberculosis and the results of the clinical studies of its substitution to date published.

Keywords: tuberculosis, calcitriol.

\section{Introducción}

La tuberculosis (TB) es una enfermedad infecciosa, transmisible, de gran importancia para la salud pública mundial. Esta patología probablemente surgió hace aproximadamente 70.000 años, y acompañó los procesos de migración de humanos de África hacia el resto del mundo ${ }^{(1)}$. Es aún una epidemia global y si no se trata puede alcanzar tasas de mortalidad hasta del 70\% para personas infectadas con baciloscopias positivas $^{(2)}$.

Durante los últimos dos siglos la tuberculosis ha causado el fallecimiento de aproximadamente un billón de personas, permaneciendo dentro de las primeras 10 causas de muerte a nivel mundial y como una significativa etiología de discapacidad pulmonar crónica ${ }^{(3)}$.

Por lo anterior, a lo largo de la historia se han diseñado múltiples estrategias para controlar esta enfermedad, aún no del todo exitosas, pese a los cambios recientes de la epidemiología mundial (tendencia a la disminución de la prevalencia) ${ }^{(3)}$. 
Todos los esfuerzos de investigación realizados con el objetivo de implementar estrategias más eficientes para el diagnóstico, seguimiento y tratamiento son necesarios en todas las latitudes y en especial en los países donde la enfermedad es endémica.

Durante los últimos años, han emergido múltiples datos tanto de la investigación clínica como básica acerca de la importancia de la vitamina D en la defensa contra esta enfermedad. A continuación, se presenta una revisión de los datos más importantes a la fecha.

\section{Tuberculosis, situación actual}

\section{Epidemiología}

Aunque la incidencia ha declinado lentamente en los últimos 15 años a una tasa de 1,5\% por año, la carga de la enfermedad es sustancial. En el año 2013 fueron reportados nueve millones de casos nuevos de tuberculosis (126 casos por 100.000 ) de los cuales el $60 \%$ se concentraron en países de alta carga de la enfermedad. Sin embargo, se estima que la tasa de detección de casos solo corresponde al 64\% del total y de manera preocupante 3.3 millones de casos no fueron diagnosticados o no reportados. En contraste, la mortalidad ha declinado sustancialmente en los últimos 20 años de 30 casos por 100.000 a 16 casos por 100.000 personas para el 2013. Para este mismo año, se estimó que cerca de 1.1 millones de personas tenían coinfección con VIH (virus de la inmunodeficiencia humana), de los cuales el $80 \%$ de los casos ocurrieron en África; las muertes de tuberculosis asociadas a VIH sumaron el $25 \%$ del total del número de muertes asociadas a tuberculosis. En el 2013, se estimaron 480.000 casos nuevos de tuberculosis resistente a múltiples fármacos a nivel mundial con aproximadamente 210.000 muertes relacionadas. Los casos de tuberculosis multirresistente se reportaron en $3,5 \%$ de casos nuevos y $20,5 \%$ de los casos con retratamiento. Para el mismo año se reportaron 550.000 casos en niños y 80.000 muertes en esta población fueron reportadas en coinfección con $\operatorname{HIV}^{(3,4)}$.

De acuerdo con datos de la Organización Mundial de la Salud en su informe mundial sobre la tuberculosis del año 2016, para el año 2015 se reportó una incidencia de TB de 10,4 millones, de los cuales $11 \%$ representaban pacientes con VIH. El 60\% de los casos se dio en India, Indonesia, China, Nigeria, Pakistán y Sudáfrica. El número estimado de nuevos casos multirresistentes fue de 480.000 y el número de muertes fue de 1,4 millones. Con este panorama, la tuberculosis continúa siendo una de las diez principales causas mundiales de muerte en $2015^{(3,4)}$.

Los factores de riesgo claves asociados con tuberculosis incluyen: pobreza, sobrepoblación, desnutrición, abuso de alcohol, infección por VIH, silicosis, enfermedad renal con necesidad de diálisis, cambios fibroapicales en la radiografía, la diabetes, el tabaquismo y la terapia inmunosupresora ${ }^{(5,6)}$. Sin embargo, el riesgo atribuible varía de acuerdo con la carga global del factor de riesgo asociado, se ha estimado de la siguiente manera: VIH (11\%), tabaquismo $(15,8 \%)$, diabetes $(7,5 \%)$, alcoholismo $(9,8 \%)$, desnutrición $(26,9 \%)$, polución del aire $(22,2 \%)^{(7)}$. Estos datos tienen implicaciones obvias en la salud pública y ponen a la luz la necesidad de integración de los servicios comunitarios y no comunitarios. Estudios de modelos, pese a sus grandes limitaciones, sugieren que la eliminación de la tuberculosis es solo probable que se logre para el año 2050, si las intervenciones diagnósticas y terapéuticas (detección temprana de casos y altas tasas de curación) son combinadas con estrategias preventivas (vacunas y tratamiento de tuberculosis latente en los dos billones de personas reservorios en pacientes de países de alta y baja carga ${ }^{(8)}$.

\section{Vitamina D}

\section{Metabolismo de la vitamina D}

La vitamina D se produce en la piel a través de la exposición a los rayos ultravioleta. Sufre un proceso de transformación de 7-dehidrocolesterol a vitamina D3 o colecalciferol. También se puede absorber a través de alimentos en forma de vitamina D3 o ergocalciferol (vitamina D2) ${ }^{(9)}$, la primera se absorbe de manera más eficiente en el intestino ${ }^{(10)}$. Ambas formas hasta este punto son inactivas biológicamente.

El colecalciferol, independientemente de su forma de adquisición, es procesado por la enzima 25-hidroxilasa en el hígado para producir 25-hidroxivitamina D (25 (OH) D). En el riñón, la 25-(OH) D es hidroxilada por la enzima 1 alfa hidroxilasa (CYP27B1) a la forma activa 1,25 dihidroxivitamina D $\left(1,25(\mathrm{OH})_{2} \mathrm{D}\right)$ también conocida como calcitriol ${ }^{(9)}$. En el riñón, la producción de $1,25(\mathrm{OH})_{2}$ D es regulada por el calcio sérico y la hormona paratiroidea ${ }^{(11)}$.

Los metabolitos de la vitamina D son transportados por proteínas de unión a la vitamina D (DBPs) aunque, en su forma libre, el calcitriol (clasificado como hormona esteroidea) actúa en receptores intracelulares de vitamina D (VDRs) para iniciar la señalización intracelular ${ }^{(12)}$. Este receptor es un factor de transcripción ligando dependiente perteneciente a la superfamilia de receptores hormonales esteroides/tiroides. El receptor retinoide $\mathrm{X}$, un receptor nuclear para el 9-cis ácido retinoico es un compañero obligado del VDR para mediar la acción de la vitamina $\mathrm{D}^{(13)}$.

Las funciones clásicas de la vitamina D son la homeostasis del calcio y el metabolismo del hueso; sin embargo, se han identificado en el organismo receptores de vitamina $D$ en otros tejidos, incluyendo el sistema inmune, de hecho todas las células del sistema inmune han demostrado expresar sus receptores, por lo cual actualmente se acepta al calcitriol como un regulador del sistema inmune $\mathrm{e}^{(14)}$. 


\section{Inmunomodulación}

Se han estudiado múltiples efectos de la vitamina D sobre el sistema inmune desde la década de los 80 . Se ha descrito que inhibe la proliferación de células T y la secreción de ciertas citocinas $^{(15,16)}$, inhibe además la transcripción de IL-2, de IFN- $\gamma$, y la producción de IL-17 por las células Th17 ${ }^{(17,18)}$. En modelos murinos se ha llegado a la conclusión de que estimula la producción de IL-4 mientras regula la producción de IL-17 e INF$\gamma$, lo cual podría tener un papel importante en la regulación de enfermedades inmunes mediadas por estas citocinas ${ }^{(19)}$.

Durante las infecciones, las células del sistema inmune innato reconocen patrones de moléculas asociadas con grupos de patógenos (PAMPs), éstos son reconocidos por los receptores tipo Toll (TLR), los cuales estimulan la activación de la enzima CYP27B1 con la subsecuente producción de 1,25 (OH $)_{2}$ D3 por las células del sistema inmune ${ }^{(20)}$. La unión de la 1,25 $(\mathrm{OH})_{2}$ D3 a sus receptores (VDR) conlleva a la expresión del gen beta defensina 4A (DEFB4A) que codifica la beta defensina 4 y el gen encargado de la expresión de catelicidina. Ambos péptidos son mediadores intracelulares de la actividad antiviral y antibacteriana ${ }^{(19,21)}$.

Además de los anteriores, otros factores antimicrobianos claves estimulados por la vitamina D incluyen radicales libres de oxígeno, óxido nítrico sintasa y la forma activa de IL-1b la cual también estimula la producción de beta defensina ${ }^{(22,23)}$.

\section{Tuberculosis y vitamina D}

Tanto la desnutrición proteínico-calórica como las deficiencias de micronutrientes generan una disfunción de la inmunidad celular, una importante medida de protección contra la tuberculosis ${ }^{(24)}$. Una vez la enfermedad se desarrolla, ésta en sí misma genera un estado catabólico que resulta en un balance negativo de nitrogenados y deficiencias de micronutrientes. Generaciones de médicos que han tratado a estos pacientes han pensado que el soporte nutricional es una medida crucial para el cuidado de los mismos. Sin embargo, ¿por qué ha sido difícil demostrar en pruebas aleatorizadas, controladas que las intervenciones nutricionales mejoran los resultados de los esquemas terapéuticos?; los datos de las revisiones sistemáticas han demostrado que, si bien no hay beneficios consistentes en los resultados específicos de tuberculosis, sí se mejora el estado nutricional de los pacientes ${ }^{(25-27)}$.

En los últimos 30 años, los investigadores han descubierto muchos papeles y mecanismos de la vitamina $\mathrm{D}$, tanto en el sistema inmune innato como en el adaptativo ${ }^{(28)}$. La vitamina D promueve la eliminación del Mycobacterium tuberculosis por medio de la destrucción medida por los macrófagos ${ }^{(29)}$. Dentro de los mecanismos puntuales, se sabe que la transcripción de la catelecidina es completamente dependiente de los niveles suficientes de vitamina $\mathrm{D}$ y tiene la capacidad de destruir de las membranas micobacterianas en el fagosoma-lisosoma en los $\operatorname{macrófagos}^{(30)}$. Lo anterior ha generado que se realicen varias pruebas de fase 2 de suplementación de vitamina D, la mayoría sin mostrar beneficio sustancial en términos de resultados asociados con la enfermedad ${ }^{(31-33)}$. Con un adecuado diseño y metodología de los estudios mencionados, el tiempo medio de conversión de cultivo de esputo fue similar entre los pacientes en los grupos placebo y de vitamina D; también fue similar la proporción de pacientes con cultivo positivo a los dos meses. ¿Esto significa que la suplementación no es de valor en el manejo de la tuberculosis pulmonar? La respuesta a la pregunta anterior aún no es clara. Si bien esta molécula estimula la destrucción mediada por macrófagos de las micobacterias, también regula esta respuesta induciendo mecanismos que inactivan algunos aspectos de la respuesta inmunológica y modulan otros. La vitamina $\mathrm{D}$ en sí misma induce mecanismos que inactivan la vitamina D y la respuesta inmune innata asociada a la misma y la inflamación ${ }^{(34)}$. En términos del sistema inmune adaptativo, la vitamina D suprime la respuesta celular Th1 que es crucial contra la defensa de las micobacterias y promueve una predominancia de una respuesta de tipo Th2 con tolerancia inmunológica, inmunidad humoral y defensa contra infecciones virales ${ }^{(35)}$.

Algunos autores han sugerido que la actividad antimicobacteriana de la vitamina $\mathrm{D}$ puede operar a nivel inicial en la implantación e ingestión del bacilo por los macrófagos alveolares y las células dendríticas ${ }^{(36)}$. En otras palabras, esto significa que esta vitamina puede ser importante para la prevención de la enfermedad sintomática; más que unas dosis dadas en momentos discretos después del desarrollo de la enfermedad activa, los niveles constantes de suficiencia pueden ser importantes para evitar la enfermedad. Esta respuesta inicial, además, es dependiente no solo de la vitamina $\mathrm{D}$, sino también de varios micronutrientes y del estado nutricional, así la optimización de la respuesta inicial puede requerir la consideración de un estado nutricional más amplio. Las pruebas clínicas de conversión del esputo pueden estar mal direccionadas si la mayor acción de la vitamina D en la enfermedad activa es mitigar la respuesta inflamatoria e inmune ${ }^{(31,32)}$. Esta función se puede evidenciar mejor a través de la restricción del daño tisular y prevención de la recaída más que con la aceleración de la conversión del esputo; sin embargo, realizar estos estudios demanda mayor tiempo, costo y son más difíciles de hacer.

En cuanto a pruebas clínicas de evaluación de los niveles de vitamina $\mathrm{D}$ en lavado broncoalveolar se cuenta con dos:

1. Cadranel y colaboradores ${ }^{(37)}$ en el año de 1988 investigaron la producción extrarrenal de vitamina D en tuberculosis en un paciente con la enfermedad, hipercalcemia y niveles plasmáticos elevados de $1,25(\mathrm{OH})_{2} \mathrm{D}$, por medio de la obtención de macrófagos alveolares y células frescas cultivadas del lavado broncoalveolar. Aquí demostraron que el metabolito activo de la vitamina $\mathrm{D}\left[1,25(\mathrm{OH})_{2} \mathrm{D}\right]$ se sintetiza antes y después de los nueve meses de una terapia antimicrobiana completa. La capacidad continua de producción de la vitamina 
D estuvo asociada con alveolitis linfocítica persistente en el paciente. La producción extrarrenal de la misma probablemente contribuyó a los niveles plasmáticos aumentados en el paciente. No obstante, no observó una correlación estrecha entre los niveles de calcio y el metabolito activo de la vitamina D. Sus hallazgos sugirieron que, aunque la producción extrarrenal de la vitamina $\mathrm{D}$ ocurre en la tuberculosis, esta anormalidad no es la principal explicación del estado alterado del metabolismo del calcio que se aprecia en estos pacientes.

2. Estos mismos autores ${ }^{(38)}$ posteriormente realizaron un estudio con el objetivo de comparar la producción extrarrenal de la vitamina $\mathrm{D}\left(1,25(\mathrm{OH})_{2} \mathrm{D}\right)$ en diferentes tipos de enfermedades granulomatosas e identificar cuáles eran las células fuente de la misma, esto por medio de la conversión del metabolito inactivo a la forma activa $\left[1,25(\mathrm{OH})_{2} \mathrm{D}\right]$ en células no cultivadas pero obtenidas del lavado broncoalveolar y mononucleares en sangre periférica de pacientes normocalcémicos con sarcoidosis y tuberculosis. La vitamina D se produjo en ambos tipos de células: células del lavado (12/12 pacientes con tuberculosis y $2 / 6$ pacientes con sarcoidosis) y mononucleares $(3 / 5$ pacientes con tuberculosis y $0 / 3$ pacientes con sarcoidosis); sin embargo, no fue así para los controles. Por otro lado, los niveles fueron significativamente mayores entre las células del lavado broncoalveolar de los pacientes con tuberculosis comparados con los pacientes de sarcoidosis $(\mathrm{P}<0,001)$. La producción del metabolito activo de la vitamina D en el lavado de los pacientes con tuberculosis se correlacionó con el número de linfocitos T CD8 pero no con otros tipos celulares. Los linfocitos $\mathrm{T}$ fueron una fuente significativa de producción de la vitamina $\mathrm{D}$, pues luego de purificadas dichas células todas mantenían esta capacidad y su producción se correlacionó estrechamente con la producción de las células no purificadas. Sus resultados soportaban la conclusión previa de que la interacción linfocito-macrófago, mediada en parte por la vitamina D activa, era un importante componente de la respuesta inmune antituberculosa exitosa.

Más recientemente, Yamshchikov y colaboradores ${ }^{(39)}$ realizaron un estudio con el objetivo primario de evaluar la relación entre los niveles séricos de vitamina D y la catelicidina en pacientes con tuberculosis activa en 95 muestras del banco sérico del estudio Tuberculosis Trials Consortium. La prevalencia encontrada de insuficiencia de vitamina D [25(OH)D $<30 \mathrm{ng} / \mathrm{ml}$ ] en pacientes con tuberculosis activa fue de $86 \%$ con una concentración media de base de $20,4 \mathrm{ng} / \mathrm{ml}$. La concentración media ( \pm SD) de la catelicidina fue de 49,5 $\pm 23,8$ $\mathrm{ng} / \mathrm{ml}$. Las mayores concentraciones se correlacionaron con el esputo positivo y un peso $>10 \%$ debajo del peso corporal ideal. Lo anterior mostrando que no se encontró que el estado sérico de la vitamina D no se relaciona con las concentraciones de la catelicidina sérica.

Zhan y colaboradores ${ }^{(40)}$, en China, realizaron un estudio en el que buscaban ver el estado de la vitamina D, la catelicidina y las citocinas (interferón gamma, IL-4 e IL-7) en los linfocitos Th en pacientes con tuberculosis pulmonary diabetes mellitus, factor de riesgo ampliamente conocido para la enfermedad. Por medio de la medición sérica de los mismos, encontraron que los pacientes con tuberculosis sin diabetes y los pacientes diabéticos con tuberculosis tenían niveles en rango de deficiencia o insuficiencia de vitamina D. Los niveles de la catelicidina y las citocinas de producción en linfocitos $\mathrm{T}$, fueron mayores en los pacientes con tuberculosis con o sin diabetes que en los pacientes controles. Nuevamente no se halló una correlación entre los niveles séricos de vitamina $\mathrm{D}$ y de catelicidina.

A la fecha no se cuenta con estudios que correlacionen la producción local (pulmonar) de citocinas antituberculosas, catelicidina y los niveles de vitamina $\mathrm{D}$ en pacientes con tuberculosis o sospecha de la enfermedad y su relación con el estado sérico de la misma $\left[25(\mathrm{OH})_{2} \mathrm{D}\right]$.

\section{Fisiopatología (estudios in-vitro)}

Uno de los mecanismos por los cuales la tuberculosis ha representado un reto en cuanto a su manejo terapéutico está relacionado con su capacidad de ser una infección crónica al inhibir la activación de macrófagos, ya que una vez el bacilo es fagocitado por los macrófagos puede inhibir la fusión de fagosomas con lisosomas, lo que permite escapar de su acción bactericida y llevar a su supervivencia intracelular ${ }^{(41)}$. Esto lo logra a través de la expresión de la proteína de recubrimiento que contiene triptófano y aspartato (TACO) dentro de los fagosomas $^{(42)}$. Precisamente en este punto de la historia natural de la tuberculosis se cree que la vitamina $D$ juega un papel importante, la activación de receptores tipo Toll (TLR) por PAMPs incrementa la expresión de CYP27B1, que convierte la $25(\mathrm{OH})$ D a su forma activa $1,25(\mathrm{OH})_{2}$ D3, la cual induce la autofagia de células fagocíticas ${ }^{(43)}$.

Por otro lado, se ha comprobado que la 1,25 $(\mathrm{OH})_{2}$ D3 inhibe la actividad de metaloproteinasas (MMPs) por lo que de esta manera disminuye las cavitaciones típicamente asociadas a la infección por Mycobacterium tuberculosis (MT) ${ }^{(44)}$.

\section{Efecto protector (estudios observacionales)}

La importancia de los niveles de vitamina D en la prevención de la tuberculosis se ha descrito con mayor fuerza desde 1985 cuando investigadores observaron que las personas residentes en Reino Unido que provenían de países con alta incidencia de infección latente por TB presentaban tasas de TB activa que incluso excedían las de sus países de origen. Este hecho se asoció a que los inmigrantes presentaban deficiencia de vitamina D que se debía, probablemente, a la disminución de exposición solar en el Reino Unido ${ }^{(45,46)}$. Desde entonces se han realizado múltiples estudios que buscan reforzar esta teoría.

Los niveles de vitamina $\mathrm{D}$ son determinados a través de la medición de $25(\mathrm{OH}) \mathrm{D}$ en plasma o suero, éste es el 
producto de la primera hidroxilación y es sintetizado en el hígado. El Instituto Norteamericano de Medicina ha clasificado de la siguiente forma los valores: 50-125 nmol/L (20$50 \mathrm{ng} / \mathrm{ml}$ ) como normal, niveles entre 30-49,9 (12-19,9 ng/ $\mathrm{ml}$ ) como inadecuados y niveles por debajo de $30 \mathrm{nmol} / \mathrm{l}$ (12 ng/ml) como deficientes mientras que niveles por encima de $125 \mathrm{nmol} / \mathrm{l}(50 \mathrm{ng} / \mathrm{ml}$ ) como potencialmente perjudiciales $^{(47)}$.

En un estudio de casos y controles realizado en el año 2013 se compararon los niveles en plasma de $25(\mathrm{OH}) \mathrm{D}$ en pacientes que recibieron tratamiento para tuberculosis respecto a pacientes que no habían presentado la enfermedad. Se encontró que los pacientes que recibieron tratamiento presentaban niveles más bajos de vitamina D $(24,7 \mathrm{ng} / \mathrm{ml}$ vs. $33,6 \mathrm{ng} / \mathrm{ml}^{(48)}$. En Corea se realizó un estudio similar de casos y controles en el que se compararon los niveles de vitamina D entre pacientes con TB y pacientes sanos. Se concluyó que los pacientes con TB tienen una mayor prevalencia de deficiencia de vitamina $\mathrm{D}$ (casos 9,86 ng/ml, IQR 7,1914,15; controles 16,03 ng/ml, IQR 12,38-20,30, P < 0,001), además los niveles de vitamina $\mathrm{D}$ se incrementaron después del tratamiento ${ }^{(49)}$.

Resultados similares se encontraron en un metanálisis publicado en el año 2015; se revisó un total de 15 estudios que incluían 1.440 casos y 2.558 controles, los resultados indicaban que valores de $25(\mathrm{OH})$ D menores a $12,5 \mathrm{nmol} / \mathrm{l}$ incrementaban de forma significativa el riesgo de tuberculosis (OR $=4,556$, IC 95\%=2,200-9,435; $13-25 \mathrm{nmol} / \mathrm{L}:$ OR $=3,797$, IC 95\% = 1,935-7,405), no se encontró asociación con niveles mayores de $51 \mathrm{nmol} / \mathrm{L}^{(50)}$. Respecto al papel de la vitamina $\mathrm{D}$ en la respuesta al tratamiento anti-TB, un estudio prospectivo realizado en Malawi con 169 pacientes encontró que no había relación entre los niveles de vitamina D respecto al desenlace del tratamiento y, por otro lado, se encontró que los fármacos anti TB, a pesar de sus efectos metabólicos no disminuían los niveles de vitamina D y, por el contrario, aumentaban sus niveles ${ }^{(51)}$.

También se ha buscado determinar los niveles de vitamina D en otros fluidos diferentes al plasma, en un estudio de casos y controles se midieron los niveles tanto en plasma como en líquido pleural en pacientes con tuberculosis y en pacientes sanos. Tuvieron en cuenta la relación entre la vitamina D y la vitamina A, ya explicada, por lo que aparte de los niveles de colecalciferol incluyeron en la medición de 13-cis ácido retinoico. Encontraron que los niveles de ambos compuestos fueron menores en pacientes con tuberculosis (niveles de colecalciferol en plasma $67,45(10,71) \mathrm{nmol} / \mathrm{L}$ y en líquido pleural $21,40(8 ., 58) \mathrm{nmol} / \mathrm{L}$ de pacientes con tuberculosis vs. pacientes sanos, plasma: $117,43(18,40) \mathrm{nmol} / \mathrm{L}(\mathrm{P}<0,001)$ y líquido pleural 94,73 $(33,34) \mathrm{nmol} / \mathrm{L}(\mathrm{P}=0,0049)$ y niveles de ácido retinoico, casos $1,51(0,72) \mathrm{nmol} / \mathrm{L}$ vs. controles $6,67(0,81)$ $\mathrm{nmol} / \mathrm{L}(\mathrm{P}<0,001))^{(52)}$.

\section{¿Profilaxis con vitamina D?}

Son pocos los estudios que han analizado el papel de la administración de vitamina $\mathrm{D}$ en la profilaxis de tuberculosis. Hasta ahora la mayor parte de la evidencia se concentra en el hecho de que en la mayoría de pacientes con tuberculosis hay niveles de vitamina D deficientes. Los estudios hasta ahora realizados en el campo de la profilaxis han utilizado la conversión de la prueba de tuberculina como método para evaluar la efectividad de la profilaxis, aquellos pacientes que presentaron conversión en esta prueba presentaban niveles significativamente menores de vitamina $\mathrm{D}^{(53,54)}$.

\section{Efecto terapéutico}

El efecto terapéutico de la vitamina D en la infección por tuberculosis no es un asunto reciente. Si bien actualmente se cuenta con estudios clínicos que ayudan a sustentar esta idea, la utilización de la vitamina D presenta una evolución histórica en el manejo de la tuberculosis.

\section{Helioterapia}

Los beneficios de la luz ultravioleta en el tratamiento de la tuberculosis cutánea se dieron a conocer en 1903 y fueron suficientes para ser motivo de entrega del premio Nobel de Medicina al Dr. Finsen. En ese mismo año, un suizo de apellido Rollier abrió las puertas de un hospital para el tratamiento de tuberculosis, usando diferentes grados de exposición solar ${ }^{(55,56)}$.

\section{Aceite de hígado de bacalao}

Fue uno de los primeros tratamientos indicados en 1770 para la tuberculosis; conociendo su contenido rico en vitamina D fue ampliamente usado en décadas posteriores. Los diferentes casos reportados hacían referencia a la resolución de síntomas, e incluso a reducción de la mortalidad de hasta el 19\% debido al uso de este aceite ${ }^{(57)}$.

\section{Ensayos clínicos}

Un ensayo clínico se realizó con el fin de determinar si el suplemento de vitamina D durante el tratamiento de TB influía en la velocidad de recuperación. En el estudio SUCCINT (Supplementary cholecalciferol in recovery from tuberculosis) se administró de forma aleatorizada a 259 pacientes con TB 600.000 UI de vitamina D3 intramuscular o placebo por dos dosis. Después de 12 semanas, los pacientes que recibieron vitamina D presentaron mayor ganancia de peso $(3,75 \mathrm{Kg}$, (IC 95\%3,16-4,34) vs. 2,61 kg (IC 95\%1,99-3,23) p=0,009), una rápida mejoría clínica y radiológica, así como una activación del sistema inmune evidenciada en la mayor secreción de IFN-gamma en respuesta a MTBs ${ }^{(31)}$.

El efecto de la vitamina D en la tuberculosis activa se ha propuesto comprobar bajo el uso conjunto con la vitamina A, como hipótesis se ha propuesto administrar ambas vitaminas de forma inhalada con el fin de llegar de forma directa al si- 
tio de infección. Sin embargo, aún faltan estudios clínicos que comprueben esta teoría ${ }^{(58)}$. En diferentes ensayos clínicos se ha visto que la suplementación con vitamina D durante la fase activa de la enfermedad acelera su resolución y permite la conversión del esputo mucho más rápido ${ }^{(59)}$.

Las dosis utilizadas varían en los diferentes estudios hasta ahora revisados. Nursyam et al. encontraron un aumento significativo en la tasa de conversión de esputo después de administrar $0,25 \mathrm{mg} /$ día de vitamina $\mathrm{D}$ durante la sexta semana de tratamiento inicial de $\mathrm{TB}^{(60)}$, Kota et al. encontraron que $60.000 \mathrm{UI} /$ semana de vitamina D y $1 \mathrm{~g} /$ día de carbonato de calcio también reducían el tiempo de conversión de esputo ${ }^{(61)}$. Sin embargo, se debe tener en cuenta que todos los estudios varían respecto a las condiciones de base de los pacientes (comorbilidades, estado de nutrición, etc.). De hecho, en un estudio en el que se administraron 100.000 UI de vitamina D solo se logró la conversión en el esputo en un grupo de pacientes que presentaban un polimorfismo en el gen del receptor de vitamina $D$, sugiriendo así la relación con factores genéticos ${ }^{(62)}$.

Como coadyuvante en el tratamiento de tuberculosis se realizó un estudio en el que a 95 pacientes que recibían manejo anti TB se les administró de forma aleatorizada altas dosis de vitamina D (2,5 mg vitamina D3 cada 15 días) vs. placebo. Los resultados demostraron que aquellos que recibieron dosis de vitamina $\mathrm{D}$ presentaron de forma más rápida conversión del esputo y lograron recuperarse de efectos secundarios del tratamiento como linfopenia, monocitosis e hipercitocinemia de una manera más rápida. De hecho, el logro que resaltan en este estudio es la supresión de citocinas proinflamatorias, las cuales se asocian a mayor riesgo de mortalidad ${ }^{(32)}$. Precisamente sobre este punto, otros estudios han evidenciado que niveles deficientes de vitamina $\mathrm{D}$ se asocian con respuesta autoinmune en pacientes con tuberculosis.
Un estudio realizado en China con 386 pacientes con tuberculosis buscó determinar la presencia de anticuerpos antinucleares (ANA) y establecer si se asociaba a deficiencia de vitamina D. Un total de 87 pacientes fueron positivos para ANAs. Se determinaron los niveles de $25(\mathrm{OH}) \mathrm{D}$ en los 87 pacientes $(\mathrm{ANA}+$ ) y en 87 pacientes (ANA-), encontrando que aquellos con anticuerpos antinucleares positivos presentaban niveles significativamente disminuidos de vitamina D $(31,3 \pm 15,1$ vs. $38,1 \pm 14,6 \mathrm{nmol} / \mathrm{l}, \mathrm{p}=0,003)$. Aunque la muestra se considera pequeña, es uno de los pocos estudios clínicos que evidencia el papel inmunomodulador de la vitamina $\mathrm{D}^{(63)}$.

\section{Conclusiones}

Como se aprecia, hay un papel claro y notable de la vitamina $\mathrm{D}$ en la fisiopatología de esta entidad infecciosa que es aún una amenaza para la salud pública global.

Pese a los resultados discordantes de los ensayos clínicos sobre la suplementación con vitamina $\mathrm{D}$ en los pacientes con tuberculosis hay varias cosas a mencionar; primero no se sabe cuál es la producción local de vitamina D a nivel pulmonar y si esta en realidad afecta la concentración de citocinas antituberculosas. Por otro lado, hay que mencionar que en algunos de estos ensayos clínicos en donde se encontraron los resultados no favorables de la suplementación se conoció el estado ulterior de los pacientes y la verdadera efectividad de la suplencia de la misma.

Se deberán esperar los datos de los estudios en curso, que esclarezcan todas las dudas que surgen de estos aspectos muy interesantes de la vitamina D en la salud y en la enfermedad y el impacto real que tendrá la suplementación de la misma como tratamiento coadyudante en el desenlace final de estos pacientes.

\section{Referencias}

1. Comas I, Coscolla M, Luo T, Borrell S, Holt KE, Kato-Maeda M, et al. Out-ofAfrica migration and Neolithic coexpansion of Mycobacterium tuberculosis with modern humans. Nature genetics. 2013;45(10):1176-82.

2. Tiemersma EW, van der Werf MJ, Borgdorff MW, Williams BG, Nagelkerke NJ. Natural history of tuberculosis: duration and fatality of untreated pulmonary tuberculosis in HIV negative patients: a systematic review. PloS one. 2011;6(4):e17601.

3. Dheda K, Barry CE, 3rd, Maartens G. Tuberculosis. Lancet (London, England). 2016;387(10024):1211-26.

4. Zumla A, George A, Sharma V, Herbert RH, Oxley A, Oliver M. The WHO 2014 global tuberculosis report--further to go. The Lancet Global health. 2015;3(1):e10-2.

5. Melsew YA, Doan TN, Gambhir M, Cheng AC, McBryde E, Trauer JM. Risk factors for infectiousness of patients with tuberculosis: a systematic review and meta-analysis. Epidemiology and infection. 2018:1-9.

6. Lawn SD, Badri M, Wood R. Tuberculosis among HIV-infected patients receiving HAART: long term incidence and risk factors in a South African cohort. AIDS (London, England). 2005;19(18):2109-16.

7. Lonnroth K, Castro KG, Chakaya JM, Chauhan LS, Floyd K, Glaziou P, et al. Tuberculosis control and elimination 2010-50: cure, care, and social development. Lancet (London, England). 2010;375(9728):1814-29.

8. Dye C, Glaziou P, Floyd K, Raviglione M. Prospects for tuberculosis elimination. Annual review of public health. 2013;34:271-86.
9. Borella E, Nesher G, Israeli E, Shoenfeld Y. Vitamin D: a new anti-infective agent? Annals of the New York Academy of Sciences. 2014;1317:76-83.

10. Trang HM, Cole DE, Rubin LA, Pierratos A, Siu S, Vieth R. Evidence that vitamin D3 increases serum 25-hydroxyvitamin D more efficiently than does vitamin D2. The American journal of clinical nutrition. 1998;68(4):854-8.

11. DeLuca HF. Overview of general physiologic features and functions of vitamin D. The American journal of clinical nutrition. 2004;80(6 Suppl):1689s-96s.

12. Gombart AF. The vitamin D-antimicrobial peptide pathway and its role in protection against infection. Future microbiology. 2009;4(9):1151-65.

13. Kliewer SA, Umesono K, Mangelsdorf DJ, Evans RM. Retinoid X receptor interacts with nuclear receptors in retinoic acid, thyroid hormone and vitamin D3 signalling. Nature. 1992;355(6359):446-9.

14. Grant WB, Holick MF. Benefits and requirements of vitamin D for optimal health: a review. Alternative medicine review : a journal of clinical therapeutic. 2005;10(2):94-111.

15. Rigby WF, Stacy T, Fanger MW. Inhibition of T lymphocyte mitogenesis by 1,25-dihydroxyvitamin D3 (calcitriol). The Journal of clinical investigation. 1984;74(4):1451-5.

16. Provvedini DM, Tsoukas CD, Deftos LJ, Manolagas SC. 1,25-dihydroxyvitamin D3 receptors in human leukocytes. Science (New York, NY). 1983;221(4616):1181-3

17. Boonstra A, Barrat FJ, Crain C, Heath VL, Savelkoul HF, O'Garra A. 1alpha,25Dihydroxyvitamin $\mathrm{d} 3$ has a direct effect on naive CD4(+) T cells to enhance 
the development of Th2 cells. Journal of immunology (Baltimore, Md : 1950). 2001;167(9):4974-80.

18. Palmer MT, Lee YK, Maynard CL, Oliver JR, Bikle DD, Jetten AM, et al. Lineagespecific effects of 1,25-dihydroxyvitamin $\mathrm{D}(3)$ on the development of effector CD4 T cells. The Journal of biological chemistry. 2011;286(2):997-1004.

19. Cantorna MT, Snyder L, Lin YD, Yang L. Vitamin D and 1,25(OH)2D regulation of T cells. Nutrients. 2015;7(4):3011-21.

20. Wang TT, Nestel FP, Bourdeau V, Nagai Y, Wang Q Liao J, et al. Cutting edge: 1,25-dihydroxyvitamin D3 is a direct inducer of antimicrobial peptide gene expression. Journal of immunology (Baltimore, Md : 1950). 2004;173(5):2909-12.

21. Gombart AF, Borregaard N, Koeffler HP. Human cathelicidin antimicrobial peptide (CAMP) gene is a direct target of the vitamin $D$ receptor and is strongly up-regulated in myeloid cells by 1,25-dihydroxyvitamin D3. FASEB journal : official publication of the Federation of American Societies for Experimental Biology. 2005;19(9):1067-77.

22. Rockett KA, Brookes R, Udalova I, Vidal V, Hill AV, Kwiatkowski D. 1,25-Dihydroxyvitamin D3 induces nitric oxide synthase and suppresses growth of Mycobacterium tuberculosis in a human macrophage-like cell line. Infection and immunity. 1998;66(11):5314-21.

23. Verway M, Bouttier M, Wang TT, Carrier M, Calderon M, An BS, et al. Vitamin D induces interleukin-1beta expression: paracrine macrophage epithelial signaling controls M. tuberculosis infection. PLoS pathogens. 2013;9(6):e1003407.

24. Cegielski JP, McMurray DN. The relationship between malnutrition and tuberculosis: evidence from studies in humans and experimental animals. The international journal of tuberculosis and lung disease : the official journal of the International Union against Tuberculosis and Lung Disease. 2004;8(3):286-98.

25. Sinclair D, Abba K, Grobler L, Sudarsanam TD. Nutritional supplements for people being treated for active tuberculosis. The Cochrane database of systematic reviews. 2011(11):Cd006086.

26. Grobler L, Nagpal S, Sudarsanam TD, Sinclair D. Nutritional supplements for people being treated for active tuberculosis. The Cochrane database of systematic reviews. 2016(6):Cd006086.

27. Lodha R, Mukherjee A, Singh V, Singh S, Friis H, Faurholt-Jepsen D, et al. Effect of micronutrient supplementation on treatment outcomes in children with intrathoracic tuberculosis: a randomized controlled trial. The American journal of clinical nutrition. 2014;100(5):1287-97.

28. Hewison M. Vitamin D and the immune system: new perspectives on an old theme. Rheumatic diseases clinics of North America. 2012;38(1):125-39.

29. Bruns H, Stenger S. New insights into the interaction of Mycobacterium tuberculosis and human macrophages. Future microbiology. 2014;9(3):327-41.

30. Aranow C. Vitamin D and the immune system. Journal of investigative medicine : the official publication of the American Federation for Clinical Research. 2011;59(6):881-6.

31. Salahuddin N, Ali F, Hasan Z, Rao N, Aqeel M, Mahmood F. Vitamin D accelerates clinical recovery from tuberculosis: results of the SUCCINCT Study [Supplementary Cholecalciferol in recovery from tuberculosis]. A randomized, placebo-controlled, clinical trial of vitamin D supplementation in patients with pulmonary tuberculosis'. BMC infectious diseases. 2013;13:22.

32. Coussens AK, Wilkinson RJ, Hanifa Y, Nikolayevskyy V, Elkington PT, Islam K, et al. Vitamin D accelerates resolution of inflammatory responses during tuberculosis treatment. Proceedings of the National Academy of Sciences of the United States of America. 2012;109(38):15449-54.

33. Ralph AP, Waramori G, Pontororing GJ, Kenangalem E, Wiguna A, Tjitra E, et al. L-arginine and vitamin D adjunctive therapies in pulmonary tuberculosis: a randomised, double-blind, placebo-controlled trial. PloS one. 2013;8(8):e70032.

34. Bhalla AK, Amento EP, Serog B, Glimcher LH. 1,25-Dihydroxyvitamin D3 inhibits antigen-induced $\mathrm{T}$ cell activation. Journal of immunology (Baltimore, Md : 1950). 1984;133(4):1748-54.

35. Penna G, Adorini L. 1 Alpha,25-dihydroxyvitamin D3 inhibits differentiation, maturation, activation, and survival of dendritic cells leading to impaired alloreactive T cell activation. Journal of immunology (Baltimore, Md : 1950). 2000;164(5):2405-11.

36. Liu PT, Stenger S, Li H, Wenzel L, Tan BH, Krutzik SR, et al. Toll-like receptor triggering of a vitamin D-mediated human antimicrobial response. Science (New York, NY). 2006;311(5768):1770-3.

37. Cadranel J, Hance AJ, Milleron B, Paillard F, Akoun GM, Garabedian M. Vitamin D metabolism in tuberculosis. Production of 1,25(OH)2D3 by cells recovered by bronchoalveolar lavage and the role of this metabolite in calcium homeostasis. The American review of respiratory disease. 1988;138(4):984-9.

38. Cadranel J, Garabedian M, Milleron B, Guillozo H, Akoun G, Hance AJ. $1,25(\mathrm{OH}) 2 \mathrm{D} 2$ production by $\mathrm{T}$ lymphocytes and alveolar macrophages recovered by lavage from normocalcemic patients with tuberculosis. The Journal of clinical investigation. 1990;85(5):1588-93.

39. Yamshchikov AV, Kurbatova EV, Kumari M, Blumberg HM, Ziegler TR, Ray SM, et al. Vitamin D status and antimicrobial peptide cathelicidin (LL-37) concentrations in patients with active pulmonary tuberculosis. The American journal of clinical nutrition. 2010;92(3):603-11.
40. Zhan Y, Jiang L. Status of vitamin D, antimicrobial peptide cathelicidin and T helper-associated cytokines in patients with diabetes mellitus and pulmonary tuberculosis. Experimental and therapeutic medicine. 2015;9(1):11-6.

41. Malik ZA, Denning GM, Kusner DJ. Inhibition of Ca(2+) signaling by Mycobacterium tuberculosis is associated with reduced phagosome-lysosome fusion and increased survival within human macrophages. The Journal of experimental medicine. 2000;191(2):287-302.

42. Anand PK, Kaul D. Downregulation of TACO gene transcription restricts mycobacterial entry/survival within human macrophages. FEMS microbiology letters. 2005;250(1):137-44.

43. Campbell GR, Spector SA. Vitamin D inhibits human immunodeficiency virus type 1 and Mycobacterium tuberculosis infection in macrophages through the induction of autophagy. PLoS pathogens. 2012;8(5):e1002689.

44. Coussens A, Timms PM, Boucher BJ, Venton TR, Ashcroft AT, Skolimowska $\mathrm{KH}$, et al. 1alpha,25-dihydroxyvitamin D3 inhibits matrix metalloproteinases induced by Mycobacterium tuberculosis infection. Immunology. 2009;127(4):539-48.

45. Davies PD. A possible link between vitamin D deficiency and impaired host defence to Mycobacterium tuberculosis. Tubercle. 1985;66(4):301-6.

46. Martineau AR. Old wine in new bottles: vitamin D in the treatment and prevention of tuberculosis. The Proceedings of the Nutrition Society 2012;71(1):84-9.

47. Ross AC, Manson JE, Abrams SA, Aloia JF, Brannon PM, Clinton SK, et al. The 2011 report on dietary reference intakes for calcium and vitamin $D$ from the Institute of Medicine: what clinicians need to know. The Journal of clinical endocrinology and metabolism. 2011;96(1):53-8.

48. Huaman MA, Sterling TR, Shepherd BE, Fiske CT. 25-Hydroxyvitamin D levels after recovery from tuberculosis: insights into pathogenesis. Tuberculosis (Edinburgh, Scotland). 2014:94(1):51-4.

49. Hong JY, Kim SY, Chung KS, Kim EY, Jung JY, Park MS, et al. Association between vitamin D deficiency and tuberculosis in a Korean population. The international journal of tuberculosis and lung disease : the official journal of the International Union against Tuberculosis and Lung Disease. 2014;18(1):73-8.

50. Zeng J, Wu G, Yang W, Gu X, Liang W, Yao Y, et al. A serum vitamin D level $<25 \mathrm{nmol} / 1$ pose high tuberculosis risk: a meta-analysis. PloS one. 2015;10(5):e0126014.

51. Sloan DJ, Mwandumba HC, Kamdolozi M, Shani D, Chisale B, Dutton J, et al. Vitamin D deficiency in Malawian adults with pulmonary tuberculosis: risk factors and treatment outcomes. The international journal of tuberculosis and lung disease : the official journal of the International Union against Tuberculosis and Lung Disease. 2015;19(8):904-11.

52. Srinivasan A, Syal K, Banerjee D, Hota D, Gupta D, Kaul D, et al. Low plasma levels of cholecalciferol and 13-cis-retinoic acid in tuberculosis: implications in host-based chemotherapy. Nutrition (Burbank, Los Angeles County, Calif). 2013;29(10):1245-51

53. Ganmaa D, Giovannucci E, Bloom BR, Fawzi W, Burr W, Batbaatar D, et al. Vitamin D, tuberculin skin test conversion, and latent tuberculosis in Mongolian school-age children: a randomized, double-blind, placebo-controlled feasibility trial. The American journal of clinical nutrition. 2012;96(2):391-6.

54. Arnedo-Pena A, Juan-Cerdan JV, Romeu-Garcia A, Garcia-Ferrer D, HolguinGomez R, Iborra-Millet J, et al. Latent tuberculosis infection, tuberculin skin test and vitamin D status in contacts of tuberculosis patients: a cross-sectional and case-control study. BMC infectious diseases. 2011;11:349.

55. Roelandts R. The history of phototherapy: something new under the sun? Journal of the American Academy of Dermatology. 2002;46(6):926-30.

56. Selvaraj P. Vitamin D, vitamin D receptor, and cathelicidin in the treatment of tuberculosis. Vitamins and hormones. 2011;86:307-25.

57. Grad R. Cod and the consumptive: a brief history of cod-liver oil in the treatment of pulmonary tuberculosis. Pharmacy in history. 2004;46(3):106-20.

58. Syal K, Chakraborty S, Bhattacharyya R, Banerjee D. Combined inhalation and oral supplementation of Vitamin A and Vitamin D: a possible prevention and therapy for tuberculosis. Medical hypotheses. 2015;84(3):199-203.

59. Turnbull ER, Drobniewski F. Vitamin D supplementation: a comprehensive review on supplementation for tuberculosis prophylaxis. Expert review of respiratory medicine. 2015;9(3):269-75.

60. Nursyam EW, Amin Z, Rumende CM. The effect of vitamin D as supplementary treatment in patients with moderately advanced pulmonary tuberculous lesion. Acta medica Indonesiana. 2006;38(1):3-5.

61. Kota SK, Jammula S, Kota SK, Tripathy PR, Panda S, Modi KD. Effect of vitamin D supplementation in type 2 diabetes patients with pulmonary tuberculosis. Diabetes \& metabolic syndrome. 2011;5(2):85-9.

62. Martineau AR, Timms PM, Bothamley GH, Hanifa Y, Islam K, Claxton AP, et al. High-dose vitamin D(3) during intensive-phase antimicrobial treatment of pulmonary tuberculosis: a double-blind randomised controlled trial. Lancet (London, England). 2011;377(9761):242-50.

63. Li W, Zhang R, Zhang K, Song L, Hong PH, Zhang Y, et al. Reduced vitamin D levels are associated with autoimmune response in tuberculosis patients. Annals of the rheumatic diseases. 2012;71(5):790. 